\title{
Anatomical configurations associated with posthemorrhagic hydrocephalus among premature infants with intraventricular hemorrhage
}

\author{
Hannah M. Tully, MD, MSc, ${ }^{1,4}$ Tara L. Wenger, MD, PhD, ${ }^{2}$ Walter A. Kukull, $\mathrm{PhD},{ }^{3}$ \\ Dan Doherty, MD, PhD, ${ }^{2,4}$ and William B. Dobyns, MD1,2,4 \\ Departments of ${ }^{1}$ Neurology, ${ }^{2}$ Pediatrics, and ${ }^{3}$ Epidemiology, University of Washington; and ${ }^{4}$ Center for Integrative Brain \\ Research, Seattle Children's Research Institute, Seattle, Washington
}

\begin{abstract}
OBJECTIVE Intraventricular hemorrhage (IVH) is a complication of prematurity often associated with ventricular dilation, which may resolve over time or progress to posthemorrhagic hydrocephalus (PHH). This study investigated anatomical factors that could predispose infants with IVH to $\mathrm{PHH}$.

METHODS The authors analyzed a cohort of premature infants diagnosed with Grade III or IV IVH between 2004 and 2014. Using existing ultrasound and MR images, the CSF obstruction pattern, skull shape, and brain/skull ratios were determined, comparing children with $\mathrm{PHH}$ to those with resolved ventricular dilation (RVD), and comparing both groups to a set of healthy controls.

RESULTS Among 110 premature infants with Grade III or IV IVH, 65 (59\%) developed PHH. Infants with PHH had more severe ventricular dilation compared with those with RVD, although ranges overlapped. Intraventricular CSF obstruction was observed in $36(86 \%)$ of 42 infants with PHH and $0(0 \%)$ of 18 with RVD $(p<0.001)$. The distribution of skull shapes in infants with $\mathrm{PHH}$ was similar to those with RVD, although markedly different from controls. No significant differences in supratentorial brain/skull ratio were observed; however, the mean infratentorial brain/skull ratio of infants with $\mathrm{PHH}$ was $5 \%$ greater (more crowded) than controls $(p=0.006)$, whereas the mean infratentorial brain/skull ratio of infants with RVD was $8 \%$ smaller (less crowded) than controls $(p=0.004)$.

CONCLUSIONS Among premature infants with IVH, intraventricular obstruction and infratentorial crowding are strongly associated with $\mathrm{PHH}$, further underscoring the need for brain MRI in surgical planning. Prospective studies are required to determine which factors are cause and which are consequence, and which can be used to predict the need for surgical intervention.
\end{abstract}

http://thejns.org/doi/abs/10.3171/2016.8.FOCUS16241

KEY WORDS posthemorrhagic hydrocephalus; intraventricular hemorrhage; prematurity

$\mathrm{I}$ NTRAVENTRICULAR hemorrhage (IVH) is a common consequence of prematurity and, when severe, may result in posthemorrhagic hydrocephalus (PHH). However, only some infants with severe IVH develop PHH; others experience ventricular dilation that resolves over time (resolved ventricular dilation; RVD). Although risk factors for IVH have been the focus of extensive research, less is known about factors that influence the progression to PHH after IVH. A better understanding of these factors could influence treatment decisions. Hydrocephalus can involve intraventricular (obstructive) or extraventricular (communicating) obstruction of CSF. ${ }^{7}$ However, the predominant pattern of CSF obstruction that develops after IVH, and how this correlates with progression or resolution, is unclear. Although PHH is most commonly treated with placement of a ventriculoperitoneal shunt, alternative procedures such as endoscopic third ventriculostomy (ETV) with choroid plexus cauterization (CPC) are available. ${ }^{5} \mathrm{ETV} / \mathrm{CPC}$ has been successfully used in many clinical settings and is believed to be particularly effective in

ABBREVIATIONS $\mathrm{CPC}=$ choroid plexus cauterization; ETV = endoscopic third ventriculostomy; IVH = intraventricular hemorrhage; $\mathrm{PHH}=$ posthemorrhagic hydrocephalus; $R V D=$ resolved ventricular dilation. 
intraventricular obstruction, especially at the level of the aqueduct. ${ }^{4}$ Identifying patterns of CSF obstruction associated with PHH could therefore allow for more targeted surgical treatment.

The optimal timing for surgical intervention after $\mathrm{PHH}$ is also uncertain. ${ }^{2}$ Having a higher threshold for surgical intervention avoids unnecessary surgery and lifelong shunt dependence for infants whose ventricular dilation would ultimately resolve over time. However, delayed treatment could be harmful to infants whose ventricular dilation is only liable to worsen. ${ }^{9}$ A better understanding of which infants are prone to develop PHH and which are likely to experience RVD could allow for earlier surgical treatment for those who need it and avoid intervention for those who do not.

Specific brain and skull configurations, particularly those that cause posterior fossa crowding, can impair normal CSF flow patterns, ${ }^{6}$ presumably through a combination of direct obstruction, altered pulsatile dynamics, and elevated venous pressures. ${ }^{1}$ Whether these configurations could act as additional contributing factors to $\mathrm{PHH}$ among infants with IVH, and whether they should be taken into account in surgical decision making, is similarly uncertain.

To investigate anatomical and physiological factors that might predispose infants with IVH to $\mathrm{PHH}$, we investigated a population of premature infants with severe IVH associated with subacute ventricular dilation. We characterized patterns of CSF obstruction, skull shape, and supra- and infratentorial brain/skull area ratios, comparing infants whose ventricular dilation resolved over time to those who developed progressive hydrocephalus. In addition, we compared both groups to a separate series of healthy age- and sex-matched controls to gauge whether differences pertained more to factors related to IVH (such as prematurity) or to outcome (PHH or RVD).

\section{Methods \\ Study Design}

We conducted a qualitative and quantitative descriptive analysis of a series of children with IVH, using existing clinical records and imaging studies. Infants with PHH were compared with those with RVD and with age- and sex-matched controls. This study was performed with the approval of the Seattle Children's Hospital Institutional Review Board. A waiver of consent was obtained because the study involved the use of existing medical records only.

\section{Study Population}

The study population consisted of premature infants with postnatal onset, prematurity-associated IVH accompanied by ventricular dilation who were treated at a tertiary care center between 2004 and 2014. Infants were initially identified through electronic medical records using ICD-9 discharge codes for intraventricular hemorrhage (772.1), and within the hospital's imaging database through the search terms related to hydrocephalus and ventricular dilation; inclusion criteria were verified through record review. Infants were excluded from analysis if IVH was prenatal in onset or not clearly related to prematurity. Infants were also excluded if they had major additional fac- tors that might have contributed to hydrocephalus (such as spina bifida).

The presence of subacute ventricular dilation-i.e., more than could be explained by acute mechanical dilation from hemorrhage, and not attributable to an ex vacuo phenomenon-was ascertained initially from official radiology reports and was subsequently verified though imaging review on the basis of qualitative findings (the presence of subjectively enlarged lateral ventricles), with review of several sequential scans when necessary. Of note, all infants in the study population had received a diagnosis of Grade III or IV IVH (for which ventricular dilation is obligatory), although this was not part of our formal inclusion criteria.

To allow comparison of age- and sex-dependent measurements, a matched control was selected for each patient (matched to corrected age within 1 month for children less than 1 year of age, within 2 months for children more than 1 and less than 2 years old, and within 6 months for children older than 2 years old.) All control scans were taken from a set of previously identified clinical MR images with normal results that had been performed on developmentally normal children (assumed to be full-term) for reasons unrelated to hydrocephalus or other major neurological problems (e.g., studies performed to assess for intracranial involvement of facial birthmarks).

\section{Outcome Definition}

Infants were considered to have PHH if they underwent a surgical procedure intended to permanently divert CSF flow (such as a ventriculoperitoneal or ventriculoatrial shunt, or ETV) or if there was no resolution of severe ventricular dilation after a year, unless dilation was ex vacuo. Infants were considered to have RVD if they had undergone no neurosurgical procedures of any kind, including external ventricular drain placement, and had subsequent imaging evidence of normal ventricular size. Infants who underwent temporizing surgical procedures, such as an extraventricular drain, without a subsequent PHH-defining surgical procedure were excluded from analysis.

\section{Data Collected}

Demographic and infant-specific information collected included date of birth, sex, ethnicity, gestational age, single versus multiple gestation, receipt of antenatal steroids, delivery method (vaginal, Cesarean section), birth weight, Apgar scores at 1 and 5 minutes, IVH grade (as recorded in medical records, verified though imaging review), sepsis (culture-proven, or presumed with a full course of antibiotic treatment administered), and meningitis.

\section{Imaging Review}

All available images were reviewed with $\mathrm{PHH} / \mathrm{RVD} /$ control status concealed, to the extent possible; some images contained views of ventriculoperitoneal shunts, precluding concealment.

\section{Maximal Ventricular Dilation}

Maximal ventricular dilation was defined as the largest ventricle size on any cranial ultrasound study, as determined by review of all available ultrasounds. Levene 

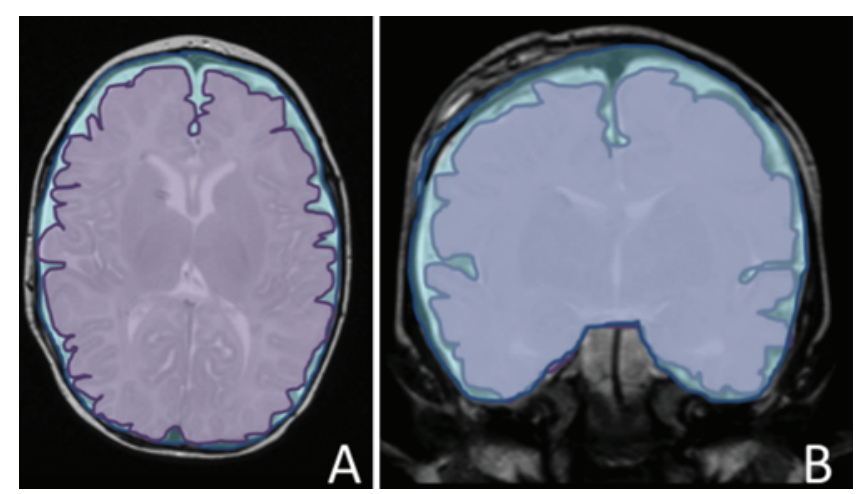

FIG. 1. Supratentorial brain/skull ratios. A: Representation of the supratentorial axial ratio, defined as the axial cross-sectional brain area (lavender) measured at closest approximation of the thalamus, divided by the axial interior skull area, measured on the same slice (blue). B: Representation of the supratentorial coronal ratio, defined as the coronal cross-sectional brain area (lavender) measured at the anterior margin of the third ventricle, on the slice with the most complete view of the basilar artery, divided by the coronal interior skull area on the same slice (b/ue). The overall supratentorial brain/skull ratio is equal to the mean of the axial and coronal ratios. These images are visual representations created using Microsoft drawing tools, designed to illustrate how quantification was performed. Actual quantification was performed with semiautomated imaging analysis software.

Index, anterior horn width, and thalamooccipital distance were measured using standard methods. ${ }^{3}$ Bilateral measurements were taken, with the larger size used in calculations.

\section{Point of CSF Obstruction}

Point of CSF obstruction was assessed qualitatively based on MR images demonstrating active ventricular dilation, i.e., progressive ventricular expansion, not explained by an ex vacuo process, present prior to surgery or at the time of shunt failure in children with $\mathrm{PHH}$, or prior to spontaneous resolution in children with RVD. The point of CSF obstruction was evaluated on the basis of presurgical MR images whenever possible; when only postsurgical MR images were available, we reviewed all presurgical CT scans and ultrasounds to make sure that pre- and post- surgical findings were consistent. Children were excluded from analysis if presurgical findings could not be assessed or were inconsistent with postsurgical findings. We classified the obstruction as intraventricular (foramen of Monro, aqueduct, fourth ventricular outflow tracts), or extraventricular (i.e., communicating hydrocephalus). Extraventricular obstruction was further classified as primarily supratentorial or infratentorial. Most MR images demonstrated a single, clear point of obstruction. If an MR image demonstrated more than 1 point of obstruction, it was categorized under the predominant point of obstruction.

\section{Overall Skull Shape and Ratios Between Brain and Skull Areas}

Overall skull shape and ratios between brain and skull areas were assessed using available MR images and compared between PHH and RVD, as well as with age- and sex-matched controls without IVH. Overall skull shape was qualitatively assigned to 1 of 5 established categories (normocephalic, brachycephalic, dolichocephalic, asymmetrical, and bitemporal narrowing) based upon midsagittal, axial, and coronal planes. We measured the cephalic index (biparietal diameter of skull/anterior-posterior length of skull $\times 100$ ) using a combination of sagittal and axial planes. We measured cross-sectional areas of the skull and brain at readily identifiable, robust landmarks that allowed for consistent measurements (Figs. 1 and 2). We measured the supratentorial skull and brain in 2 planes: at the closest approximation of the thalamus in the axial plane; and at the anterior margin of the third ventricle, measured on the slice that offered the most complete view of the basilar artery, in the coronal plane. We measured cross-sectional areas of the cerebellum and posterior fossa in 3 planes: at the midline cerebellar vermis in the sagittal plane, at the straight sinus just beyond the confluence of the cerebral veins in the coronal plane, and at the caudal boundary of the fourth ventricle in the axial plane. We calculated the ratio of cross-sectional areas of brain to skull in each plane, as well as the mean ratio for supratentorial and infratentorial structures. Mean ratios were only calculated if all individual planes had been successfully measured.

All linear measurements were performed directly on scans using a picture archiving and communication sys-
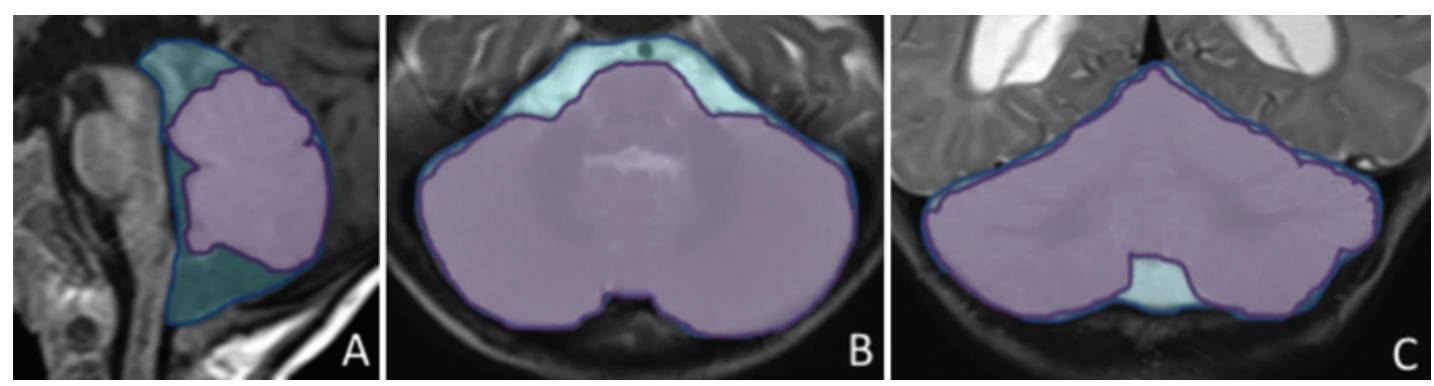

FIG. 2. Infratentorial brain/skull ratios. A: Representation of the infratentorial sagittal ratio, defined as the sagittal cross-sectional cerebellar vermis area (lavender) at the midline vermis, divided by the coronal interior skull area (blue) measured on the same slice. B: Representation of the infratentorial axial ratio, defined as the axial cross-sectional cerebellar area (lavender) measured at the caudal border of the fourth ventricle divided by the axial interior skull area (blue) on the same slice. C: Representation of the infratentorial coronal ratio, defined as the coronal cross-sectional cerebellar area (lavender) measured at the straight sinus just beyond the confluence of the cerebral veins, divided by the coronal interior skull area (blue) on the same slice. These images are visual representations created using Microsoft drawing tools, designed to illustrate how quantification was performed. Actual quantification was performed with semiautomated imaging analysis software. 
TABLE 1. Clinical characteristics of infants with IVH, with and without $\mathrm{PHH}$

\begin{tabular}{|c|c|c|}
\hline Variable & $\mathrm{PHH}$ & RVD \\
\hline No. of patients & 65 & 44 \\
\hline Males (\%) & $33(50.8)$ & $27(61.4)$ \\
\hline $\begin{array}{l}\text { Mean gestational age in wks } \pm \text { SD } \\
\text { (range) }\end{array}$ & $\begin{array}{r}27.2 \pm 3.0 \\
(22-36)\end{array}$ & $\begin{array}{r}27.1 \pm 3.8 \\
(23-36)\end{array}$ \\
\hline Multiple gestations (\%) & $14(21.5)$ & $7(15.9)$ \\
\hline \multicolumn{3}{|l|}{ Ethnicity (\%) } \\
\hline White & $39(60.0)$ & $30(69.8)$ \\
\hline Black & $6(9.2)$ & $0(0.0)$ \\
\hline Hispanic & $12(18.5)$ & $6(14.0)$ \\
\hline Asian & $2(3.1)$ & $1(2.3)$ \\
\hline Mixed & $3(4.6)$ & $3(7.0)$ \\
\hline Other/did not specify & $3(4.6)$ & $3(7.0)$ \\
\hline Antenatal steroids (\%) & $24(51.1)$ & $23(63.4)$ \\
\hline C-section delivery (\%) & $36(60.0)$ & $23(56.1)$ \\
\hline $\begin{array}{l}\text { Mean birth weight in grams } \pm \text { SD } \\
\text { (range) }\end{array}$ & $\begin{array}{l}1092 \pm 478 \\
\quad(500-2940)\end{array}$ & $\begin{array}{l}1020 \pm 550 \\
(438-2780)\end{array}$ \\
\hline \multicolumn{3}{|l|}{ Mean Apgar score \pm SD (range) } \\
\hline $1 \mathrm{~min}$ & $\begin{array}{c}3.4 \pm 2.0 \\
(0-8)\end{array}$ & $\begin{array}{c}3.5 \pm 2.4 \\
(0-8)\end{array}$ \\
\hline $5 \min$ & $\begin{array}{c}5.8 \pm 1.9 \\
(1-9)\end{array}$ & $\begin{array}{c}5.6 \pm 2.1 \\
(1-9)\end{array}$ \\
\hline \multicolumn{3}{|l|}{ IVH grade (\%) } \\
\hline 3 & $31(50.0)$ & $25(58.0)$ \\
\hline 4 & $31(50.0)$ & $18(41.9)$ \\
\hline Sepsis (\%) & $32(54.2)$ & $22(48.8)$ \\
\hline Meningitis (\%) & $5(8.1)$ & $3(7.0)$ \\
\hline
\end{tabular}

Values totaling less than the group total reflect missing data.

tem viewer (GE Centricity). All area measurements were performed on images uploaded into ImageJ open-source software (https://imagej.nih.gov/ij/), using semiautomated pixel selection with manual correction.

\section{Statistical Analysis}

We assessed differences in distributions of categorical variables (skull shape, point of CSF obstruction) and between means of continuous variables (cephalic index, brain/skull area ratios) using Student t-tests and Fisher's exact tests, as appropriate. Because this analysis is exploratory, we did not make adjustments for multiple comparisons. All statistical analyses were performed using Stata (version 14, StataCorp LP).

\section{Results}

We identified 110 infants with prematurity-associated Grade III or IV IVH; of whom 65 developed PHH (all but 1 of whom underwent surgery) and 44 experienced RVD (1 could not be classified due to insufficient follow-up). Compared with infants with RVD, infants with $\mathrm{PHH}$ were more likely to be female, more likely to be the product of multiple gestations, and less likely to have received antenatal steroids. Gestational ages, delivery method, birth
TABLE 2. Characteristics of ventricular dilation (on more dilated side) noted in infants with IVH, with and without PHH

\begin{tabular}{lcc}
\hline \multicolumn{1}{c}{ Characteristic } & PHH & RVD \\
\hline $\begin{array}{l}\text { No. of patients w/ ultrasounds avail- } \\
\text { able for quantification }\end{array}$ & 44 & \multicolumn{1}{l}{40} \\
\hline $\begin{array}{l}\text { Levene Index }>4 \text { mm above 97th } \\
\text { percentile for gestational age (\%) }\end{array}$ & $43(97.7)$ & $10(26.3)^{*}$ \\
\hline $\begin{array}{l}\text { Mean anterior horn width in } \mathrm{mm} \pm \mathrm{SD} \\
\text { (range) }\end{array}$ & $18.3 \pm 4.6$ & $7.7 \pm 4.9$ \\
Mean thalamooccipital distance in mm & $37.7 \pm 7.4$ & $24.5 \pm 7.8$ \\
\pm SD (range) & $(22-60)$ & $(12-45)$ \\
\hline Mean age at maximal dilation in days & $36.6 \pm 47.8$ & $27.0 \pm 27.6$ \\
\pm SD (range) & $(12-307)$ & $(3-146)$ \\
\hline Mean age at first surgery in days \pm SD & $41.1 \pm 49.4$ & - \\
(range) & $(15-307)$ & \\
\hline
\end{tabular}

* Only 38 patients available.

weights, and Apgar scores were similar between infants with and without PHH (Table 1).

Eighty-four infants underwent ultrasounds that could be used to quantify the degree of maximal ventricular dilation. Infants with $\mathrm{PHH}$ had more severe ventricular dilation than infants without, but ranges overlapped (Table 2). Mean age at maximal dilation was similar between groups, and the range was broad in both.

Sixty infants underwent MRI at the time of active ventricular dilation, which allowed us to qualitatively evaluate the apparent point of CSF obstruction (Fig. 3). More than half of infants with $\mathrm{PHH}$ demonstrated fourth-ventricle outlet obstruction, and a third had aqueductal obstruction (Table 3). In contrast, no infants with RVD had intraventricular obstruction; instead, all had increased extraaxial fluid (in addition to ventricular dilation). Three infants with PHH had severe posterior fossa crowding, 2 of whom had unrecognized craniosynostosis. One infant with RVD also had posterior fossa crowding and was recognized to have sagittal craniosynostosis shortly after birth, which was treated surgically at 13 months. This patient did not require additional surgery to address ventricular dilation.

Thirty-six children underwent MRI after their ventricular dilation had resolved, which we used to evaluate skull shape and brain/skull ratios (Fig. 4). We found no significant difference in overall head shape and cephalic index between infants with PHH and those with RVD (Table 4). The mean supratentorial brain/skull ratio was 3\% higher (more crowded), and the mean infratentorial ratio was $15 \%$ higher in infants with PHH compared with those with RVD, differences that were highly statistically significant. These differences remained highly significant even after we excluded the 3 patients with craniosynostosis from the analysis.

We also compared skull shape and brain/skull ratios of these 36 children to healthy controls, matched by age and sex to each child with IVH. Patients with PHH and RVD had a much broader range of skull shapes compared with their respective controls (Table 5). The mean supratentorial brain/skull ratios in infants with $\mathrm{PHH}$ and with RVD were not statistically different from their respective con- 

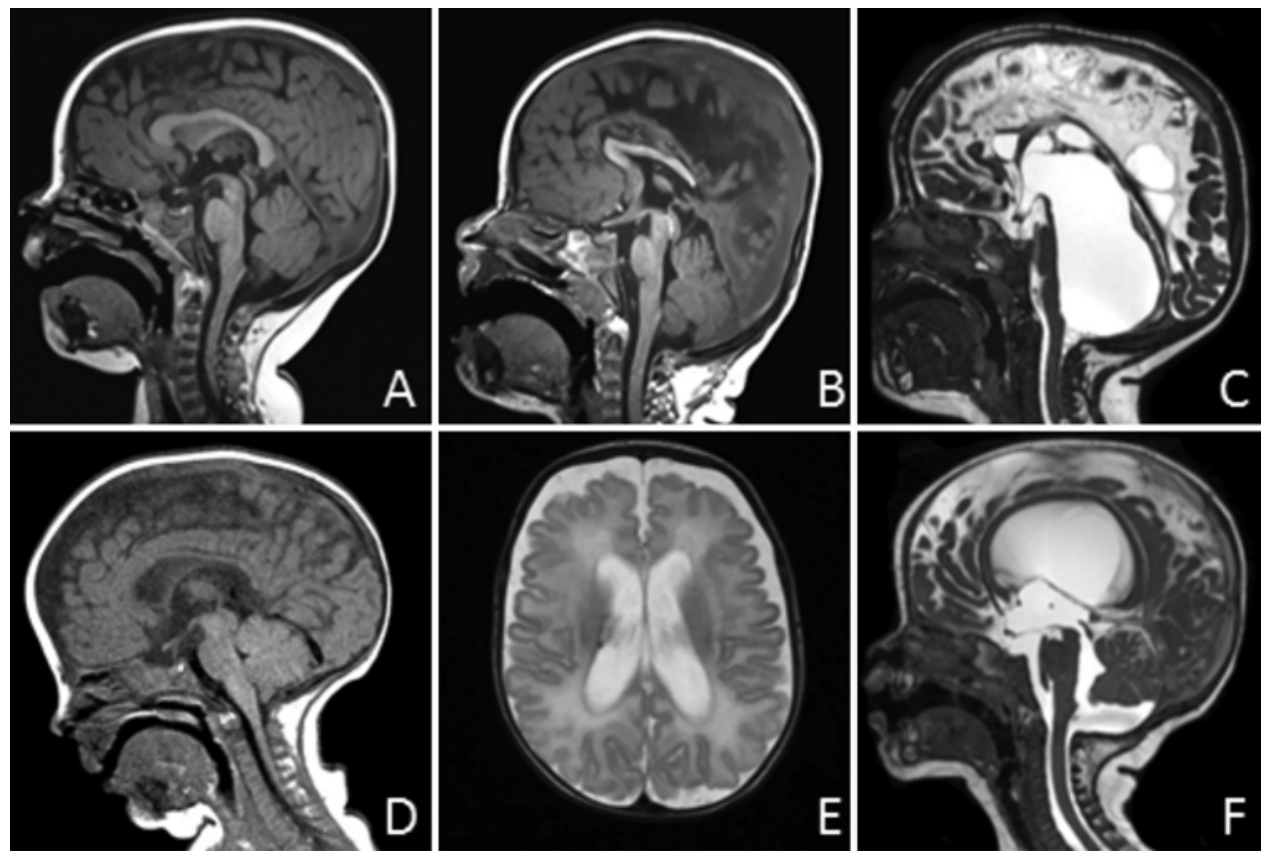

FIG. 3. CSF obstruction pattern on MRI. A: Normal anatomy. Midsagittal T1-weighted image demonstrating normal anatomy with no CSF obstruction. B: Aqueduct obstruction. Midsagittal T1-weighted image with obstruction at the level of the aqueduct, here visible as focal T1 hyperintensity. This is accompanied by dilation of the lateral ventricles (not shown in this view) and a normal-sized fourth ventricle. C: Fourth ventricle outlets. Midsagittal fast imaging employing steady-state acquisition-C/constructive interference in steady state image demonstrating massive dilation of the fourth ventricle, with the floor of the posterior fossa occupied by stretched cerebellum. This pattern may or may not be accompanied by aqueductal obstruction. D: Posterior fossa crowding. Midsagittal T1-weighted image demonstrating tight posterior fossa with reduced size of the prepontine cistern and fourth ventricle, and protrusion of the cerebellar tonsils below the level of the foramen magnum. This infant also had primary sagittal synostosis. E: Supratentorial extraaxial space. Axial T2-weighted image showing expanded extraaxial space not explained by atrophy, with no intraventricular obstruction detected on other views. This pattern is accompanied by variable expansion of the infratentorial space, although this is less prominent than the expanded supratentorial space. F: Infratentorial extraaxial space. Midsagittal T2-weighted image demonstrating expanded extraaxial space beneath the cerebellum, with modest enlargement of the fourth ventricle, but with no evidence of fourth ventricle outlet obstruction. This pattern is accompanied by variable expansion of the supratentorial extraaxial space, although this is less prominent than the expanded infratentorial space.

trol scans. However, the mean infratentorial brain/skull ratio of infants with $\mathrm{PHH}$ was 5\% greater (more crowded) than their controls, and the mean infratentorial brain/skull ratio of infants with RVD was $8 \%$ smaller (less crowded) than their controls, differences that were highly statistically significant.

\section{Discussion}

We assessed a group of 110 infants with severe prematurity-associated IVH, comparing infants with nonresolving ventricular dilation $(\mathrm{PHH})$ to those whose ventricular dilation resolved over time (RVD). Although infants with PHH had greater maximal ventricular dilation on average than infants with RVD, the ranges overlapped. Notably, one-quarter of infants with RVD had a Levene Index greater than $4 \mathrm{~mm}$ above the 97th percentile, considered a traditional threshold for surgical intervention. ${ }^{3}$

Strikingly, the majority of infants with $\mathrm{PHH}$ demonstrated intraventricular obstruction. Fourth-ventricle outlet obstruction was particularly common, noted in more than half of patients with $\mathrm{PHH}$. The fourth ventricle was not usually entrapped; in most infants, the aqueduct was visibly open on MRI, and the fourth ventricle decompressed along with the lateral ventricles after surgery. In contrast, no infants with RVD had intraventricular obstruction; all had communicating hydrocephalus.

The preponderance of intraventricular obstruction among infants with PHH carries important clinical implications, because it suggests that some infants with IVH might be particularly good candidates for ETV/CPC. Although ventriculoperitoneal shunt placement is generally considered the definitive permanent treatment for PHH in

TABLE 3. Pattern of CSF obstruction in infants with IVH, with and without $\mathrm{PHH}$

\begin{tabular}{lclll}
\hline \multicolumn{1}{c}{ Variable } & PHH $(\%)$ & RVD $(\%)$ & p Value \\
\hline No. of patients $^{*}$ & 42 & 18 & \\
\hline Aqueduct & $14(33.3)$ & $0(0.0)$ & \\
\hline Posterior fossa (crowding) & $3(7.1)$ & $1(5.6)$ & \\
\hline Fourth ventricle outlets & $22(52.4)$ & $0(0.0)$ & $<0.001 \dagger$ \\
\hline Supratentorial extraaxial space & $0(0.0)$ & $9(50.0)$ & \\
\hline Infratentorial extraaxial space & $3(7.1)$ & $8(44.4)$ & \\
\hline
\end{tabular}

* Limited to infants with MRI performed at the time of active ventricular dilatation. Reflects number of MR images available for quantification rather than total study participants.

$\dagger p$ value for homogeneity of distribution. 

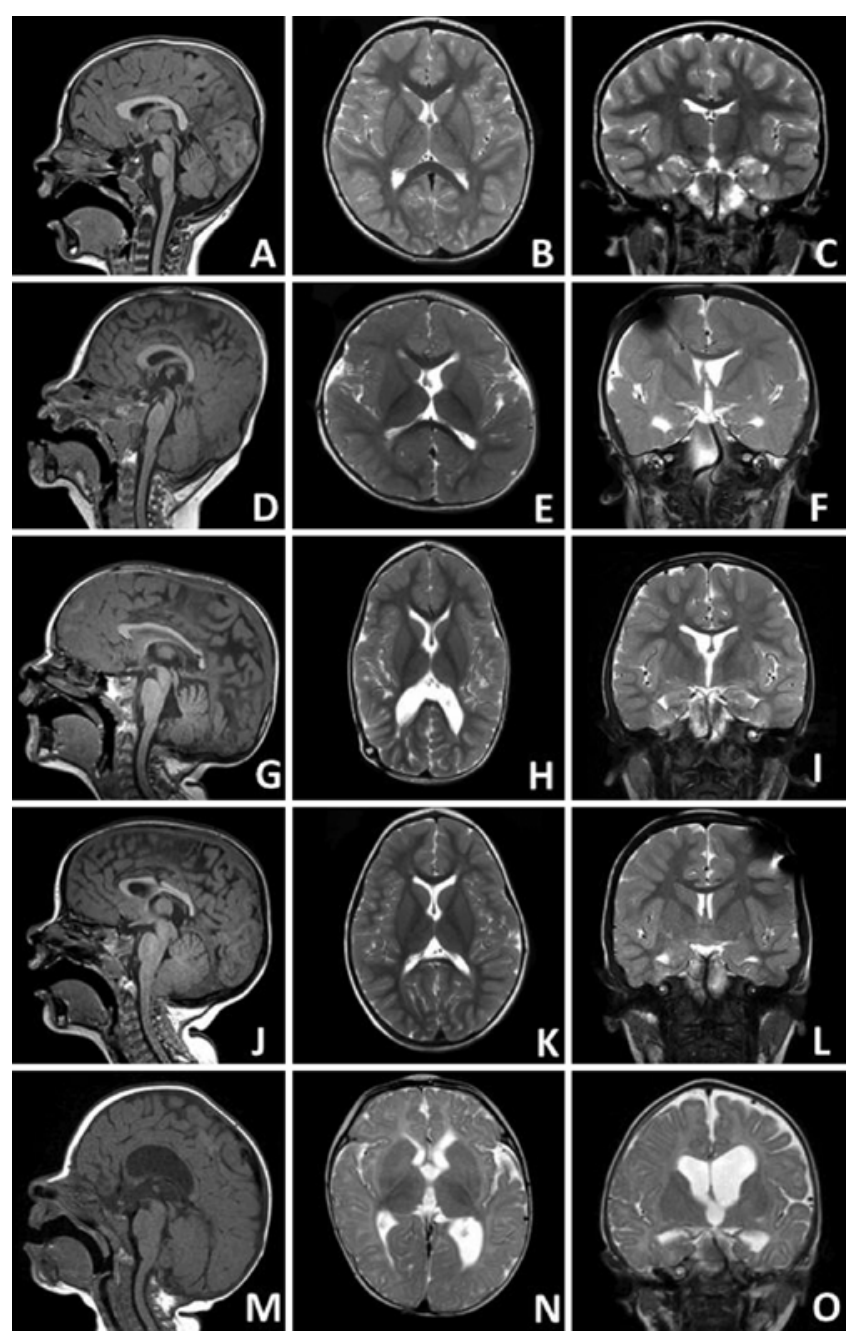

FIG. 4. Skull shapes in sagittal T1 (left column), axial T2 (center column), and coronal T2 (right column) MR images. A-C: Normocephalic. D-F: Brachycephalic, characterized by shortened anteroposterior diameter relative to biparietal diameter, particularly in rostral axial views. G-I: Dolichocephalic, characterized by shortened biparietal diameter relative to anteroposterior diameter. J-L: Bitemporal narrowing, characterized by narrow frontal diameter relative to occipital diameter in the axial plane. This patient also had significant posterior fossa crowding (note the cerebellar tonsils crowding the foramen magnum). M-0: Asymmetrical/irregular, characterized by marked asymmetry or irregularity.

this patient population, ETV/CPC can be effective ${ }^{10}$ and could potentially be used more widely, especially if the CSF obstruction pattern is taken into account in surgical planning. In addition to the patterns of CSF obstruction described in this paper, the status of the prepontine cistern should also be considered, because scarring of that cistern is associated with a higher risk of ETV/CPC failure..$^{10}$

Infants with PHH and with RVD had a much broader range of skull shapes compared with their respective controls, but not when the groups were compared with each other. This suggests that differences in overall skull shape may primarily reflect acquired changes related to prematurity, IVH, or neurodevelopmental disability.

In contrast, when we compared the brain/skull ratios
TABLE 4. Head shape and brain/skull ratios in infants with PHH compared to infants with RVD

\begin{tabular}{lccc}
\hline \multicolumn{1}{c}{ Variable } & PHH & RVD & p Value \\
\hline No. of patients* & 18 & 18 & \\
\hline Head shape & & & 0.91 \\
\hline Normocephalic & $5(27.8)$ & $7(38.9)$ & \\
\hline Brachycephalic & $4(22.2)$ & $3(16.7)$ & \\
\hline Dolichocephalic & $4(22.2)$ & $5(27.8)$ & \\
\hline Bitemporal narrowing & $1(5.6)$ & $1(5.6)$ & \\
\hline Asymmetric/irregular & $4(22.2)$ & $2(11.1)$ & \\
\hline $\begin{array}{l}\text { Mean cephalic index } \pm \text { SD } \\
\text { (range) }\end{array}$ & $(69.7 \pm 7.1$ & $81.9 \pm 6.9$ & 0.36 \\
\hline Mean supratentorial brain/ & $0.93 \pm 0.024$ & $0.90 \pm 0.045$ & 0.008 \\
skull ratio \pm SD (range) & $(0.90-0.99)$ & $(0.83-0.96)$ & \\
\hline Mean infratentorial brain/ & $0.87 \pm 0.061$ & $0.72 \pm 0.093$ & $<0.0001$ \\
skull ratio \pm SD (range) & $(0.76-0.97)$ & $(0.58-0.86)$ & \\
\hline
\end{tabular}

* Limited to infants with MRI performed after resolution of ventricular dilatation. Reflects number of MR images available for quantification rather than total study participants.

of infants with PHH and with PVD to each other and to age- and sex-matched controls, we found highly statistically significant differences in infratentorial brain/skull ratio (i.e., posterior fossa crowdedness). Infants with $\mathrm{PHH}$ had more posterior fossa crowding compared with infants with RVD and with controls; infants with RVD had less. The reason for these differences-and whether they are cause or consequence-is unclear. Among patients with RVD, increased space in the posterior fossa could be due to chronic, low-level accumulation of CSF in the subarachnoid space due to decreased absorption. Alternatively, it could be due to reduced cerebellar size as a result of prematurity, IVH, or both. ${ }^{8}$ Increased infratentorial space could also be an innate anatomical characteristic independent of prematurity and IVH. If so, it could conceivably mitigate the pathological alterations of CSF flow and increased venous pressures observed after IVH, thereby exerting a protective effect.

The increased posterior fossa crowding noted in patients with PHH is harder to explain as a direct consequence of either IVH or prematurity. It could be the result of altered CSF flow dynamics after ventriculoperitoneal shunting, although no comparable effect was observed in the supratentorial compartment. Alternatively, it could be an innate anatomical characteristic, independent of prematurity, IVH, or surgical treatment. If so, reduced space within the posterior fossa could conceivably act as a compounding risk factor for $\mathrm{PHH}$ after IVH.

Although highly statistically significant, differences in infratentorial brain/skull ratios were relatively subtle on qualitative MRI review. However, severe posterior fossa crowding was observed in 3 patients with $\mathrm{PHH}, 2$ of whom we discovered to have unrecognized craniosynostosis. One patient with RVD and pronounced posterior fossa crowding had been diagnosed with sagittal synostosis shortly after birth, which was corrected surgically at 13 months of age. Therefore, the presence of posterior fossa crowding in an infant with IVH and ventricular dilation should prompt 
TABLE 5. Head shape and brain/skull ratios compared with age- and sex-matched controls

\begin{tabular}{|c|c|c|c|c|c|c|}
\hline Variable & $\mathrm{PHH}^{*}$ & $\mathrm{PHH}$ Controls & p Value & RVD* & RVD Controls & p Value \\
\hline Skull shape & & & 0.006 & & & 0.001 \\
\hline Normocephalic & $5(27.8)$ & $15(83.3)$ & & $7(38.9)$ & $17(94.4)$ & \\
\hline Brachycephalic & $4(22.2)$ & $2(11.1)$ & & $3(16.7)$ & $0(0.0)$ & \\
\hline Dolichocephalic & $4(22.2)$ & $1(5.6)$ & & $5(27.8)$ & $0(0.0)$ & \\
\hline Bitemporal narrowing & $1(5.6)$ & $0(0.0)$ & & $1(5.6)$ & $0(0.0)$ & \\
\hline Asymmetric/irregular & $4(22.2)$ & $0(0.0)$ & & 2 (11.1) & $1(5.6)$ & \\
\hline $\begin{array}{l}\text { Mean cephalic index } \pm \text { SD } \\
\quad \text { (range) }\end{array}$ & $\begin{array}{r}79.7 \pm 7.1 \\
\quad(66-99)\end{array}$ & $\begin{array}{r}82.7 \pm 6.3 \\
\quad(71-93)\end{array}$ & 0.20 & $\begin{array}{r}81.9 \pm 6.9 \\
(71-98)\end{array}$ & $\begin{array}{r}81.3 \pm 6.8 \\
(71-98)\end{array}$ & 0.79 \\
\hline $\begin{array}{l}\text { Mean supratentorial B:S } \\
\text { ratio } \pm \text { SD (range) }\end{array}$ & $\begin{array}{l}0.93 \pm 0.024 \\
\quad(0.90-0.99)\end{array}$ & $\begin{array}{l}0.93 \pm 0.033 \\
\quad(0.86-0.97)\end{array}$ & 0.73 & $\begin{array}{r}0.90 \pm 0.045 \\
\quad(0.83-0.96)\end{array}$ & $\begin{array}{l}0.92 \pm 0.031 \\
\quad(0.83-0.96)\end{array}$ & 0.10 \\
\hline $\begin{array}{l}\text { Mean infratentorial B:S } \\
\text { ratio } \pm S D \text { (range) }\end{array}$ & $\begin{array}{r}0.87 \pm 0.061 \\
\quad(0.76-0.97)\end{array}$ & $\begin{array}{l}0.82 \pm 0.050 \\
\quad(0.73-0.91)\end{array}$ & 0.006 & $\begin{array}{l}0.72 \pm 0.093 \\
\quad(0.58-0.86)\end{array}$ & $\begin{array}{r}0.80 \pm 0.058 \\
(0.67-0.89)\end{array}$ & 0.004 \\
\hline
\end{tabular}

There were 18 participants in each group.

* Limited to infants with MRI performed after resolution of ventricular dilatation. Reflects number of MR images available for quantification rather than total study participants.

evaluation for craniosynostosis, because this could be an additional contributing factor that would change surgical management.

The major strength of this study is the amount of detailed clinical and imaging data available for analysis, which allowed us to create a cohort of "typical preemies" that could be evaluated in depth. However, this study has several limitations. Although this study investigates anatomical risk factors for $\mathrm{PHH}$, the inextricable link between PHH and its treatment meant that we assessed our outcome primarily through a proxy measure: CSF diversion surgery. Multiple elements influence the decision to proceed with surgery, including ventricle size, rate of progression of ventricular dilation, clinical signs and symptoms of raised intracranial pressure, and the preferences of parents, neonatal intensive care unit attending physicians, and consulting neurosurgeons. These elements are often not fully documented in medical records, are difficult to quantify, and are not accounted for in this analysis. These factors could influence our results in unexpected ways and deserve further investigation.

Moreover, our reliance on surgery as a proxy for $\mathrm{PHH}$ means that some infants classified as having PHH might have eventually experienced resolution had they not undergone surgery. Misclassifying infants as having PHH when they might actually have had RVD could bias our results; however, the direction of bias would likely attenuate rather than exaggerate the differences we observed between groups.

This study is also limited by its small sample size and reliance on existing data. To analyze CSF obstruction pattern, we used images performed prior to surgery or resolution of ventricular dilation. These imaging studies were obtained for a variety of clinical reasons (seizures, possible ischemia, and to evaluate ventricular dilation itself), and their results could have influenced the decision to proceed with surgery. The association between intraventricular obstruction and PHH may therefore be partially self-reinforcing (i.e., infants found to have intraventricular obstruction may have been more likely to undergo sur- gery), which could provide an additional explanation for the strong association we observed between obstruction pattern and outcome.

Although volumetric analyses of skull and brain ratios would have been optimal, not all available scans provided volumetric data. Instead, we relied upon 2D measures of anatomy, taking the average of several planes to better approximate a volumetric analysis. Scans were also performed at varying ages, and mean age differed by group. To address this potential source of bias, we compared each group with age-matched controls for skull shape and brain/skull ratios, which may vary with age.

The temporal relationship between obstruction of CSF flow and the onset of PHH is clear, but the relationship between PHH and skull shape is not. IVH is usually a perinatal event, with ventricular dilation developing shortly thereafter. No MR images that preceded the onset of IVH or ventricular dilation were available for review. Moreover, because active ventricular dilation could have confounded our estimates of crowdedness, we relied upon even later studies, performed after dilation had resolved, to assess skull shape and brain/skull ratios. The differences we observed were highly statistically significant and are worthy of further exploration. However, the design of the current study eliminates our ability to infer causality. A larger, prospectively collected data set should allow for clarification about whether the anatomical differences we observed in this study are cause or consequence of $\mathrm{PHH}$ or its treatment.

\section{Conclusions}

Among premature infants with IVH, $\mathrm{PHH}$ is strongly associated with intraventricular obstruction, particularly at the aqueduct and fourth ventricle outlets. Infratentorial crowding is also associated with $\mathrm{PHH}$, but whether this is cause or consequence remains unclear. Marked infratentorial crowding should prompt an evaluation for craniosynostosis, which may go unrecognized in the setting of prematurity and IVH, and may act as an additional risk factor 
for PHH. These findings further underscore the need for MRI in surgical planning. The interplay between CSF obstruction and posterior fossa crowding, and whether these factors can be used prospectively to determine the optimal type and timing of surgical treatment, deserves further investigation.

\section{Acknowledgments}

Funding for this study was provided by a Child Neurology Foundation Shields Award (to Dr. Tully) and by the National Center For Advancing Translational Sciences of the NIH (award no. KL2TR000421, to Dr. Tully via the University of Washington).

\section{References}

1. Bergsneider M: Evolving concepts of cerebrospinal fluid physiology. Neurosurg Clin N Am 12:631-638, vii, 2001

2. Brouwer AJ, Brouwer MJ, Groenendaal F, Benders MJ, Whitelaw A, de Vries LS: European perspective on the diagnosis and treatment of posthaemorrhagic ventricular dilatation. Arch Dis Child Fetal Neonatal Ed 97:F50-F55, 2012

3. Brouwer MJ, de Vries LS, Pistorius L, Rademaker KJ, Groenendaal F, Benders MJ: Ultrasound measurements of the lateral ventricles in neonates: why, how and when? A systematic review. Acta Paediatr 99:1298-1306, 2010

4. Kulkarni AV, Drake JM, Kestle JR, Mallucci CL, Sgouros S, Constantini S: Predicting who will benefit from endoscopic third ventriculostomy compared with shunt insertion in childhood hydrocephalus using the ETV Success Score. J Neurosurg Pediatr 6:310-315, 2010

5. Mazzola CA, Choudhri AF, Auguste KI, Limbrick DD Jr, Rogido M, Mitchell L, et al: Pediatric hydrocephalus: systematic literature review and evidence-based guidelines. Part 2: Management of posthemorrhagic hydrocephalus in premature infants. J Neurosurg Pediatr 14 (Suppl 1):8-23, 2014

6. Shaffer N, Martin B, Loth F: Cerebrospinal fluid hydrodynamics in type I Chiari malformation. Neurol Res 33:247260, 2011

7. Strahle J, Garton HJ, Maher CO, Muraszko KM, Keep RF, $\mathrm{Xi} \mathrm{G}$ : Mechanisms of hydrocephalus after neonatal and adult intraventricular hemorrhage. Transl Stroke Res 3 (Suppl 1):25-38, 2012
8. Tam EW, Miller SP, Studholme C, Chau V, Glidden D, Poskitt KJ, et al: Differential effects of intraventricular hemorrhage and white matter injury on preterm cerebellar growth. J Pediatr 158:366-371, 2011

9. van Zanten SA, de Haan TR, Ursum J, van Sonderen L: Neurodevelopmental outcome of post-hemorrhagic ventricular dilatation at 12 and 24 months corrected age with highthreshold therapy. Eur J Paediatr Neurol 15:487-492, 2011

10. Warf BC, Campbell JW, Riddle E: Initial experience with combined endoscopic third ventriculostomy and choroid plexus cauterization for post-hemorrhagic hydrocephalus of prematurity: the importance of prepontine cistern status and the predictive value of FIESTA MRI imaging. Childs Nerv Syst 27:1063-1071, 2011

\section{Disclosures}

The authors report no conflict of interest concerning the materials or methods used in this study or the findings specified in this paper.

\section{Author Contributions}

Conception and design: Tully, Wenger, Kukull, Dobyns. Acquisition of data: Tully, Wenger. Analysis and interpretation of data: Tully, Doherty. Drafting the article: Tully. Critically revising the article: all authors. Reviewed submitted version of manuscript: all authors. Approved the final version of the manuscript on behalf of all authors: Tully. Statistical analysis: Tully.

\section{Supplemental Information Previous Presentations}

Portions of this work will be presented at the 8th Annual Meeting of the International Society of Hydrocephalus and Cerebrospinal Fluid Disorders, Cartagena, Colombia, October 8-10, 2016.

\section{Correspondence}

Hannah Tully, Division of Pediatric Neurology, Seattle Children's Hospital, 4800 Sand Point Way, Seattle, WA 98115. email: hmtully@uw.edu. 